\title{
New perspectives on the approach to patients with atypical Hemolytic Uremic Syndrome candidates for renal transplantation
}

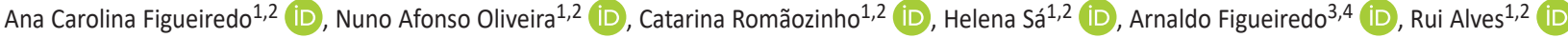 \\ ${ }^{1}$ Serviço de Nefrologia, Centro Hospitalar e Universitário de Coimbra, Coimbra, Portugal \\ ${ }^{2}$ Clínica Universitária de Nefrologia, Faculdade de Medicina da Universidade de Coimbra, Coimbra, Portugal \\ ${ }^{3}$ Serviço de Urologia e Transplantação Renal, Centro Hospitalar e Universitário de Coimbra, Coimbra, Portugal \\ ${ }^{4}$ Faculdade de Medicina da Universidade de Coimbra, Coimbra, Portugal
}

\section{ABSTRACT}

Atypical hemolytic uremic syndrome (aHUS) is one of the most challenging diseases for a nephrologist, with high rates of progression to end-stage kidney disease (ESKD) and post-transplant recurrence. Complement dysregulation has been found in up to $70 \%$ of cases, which can be hereditary or acquired. Over the last few years, knowledge of the pathogenesis of aHUS has greatly increased, with the unravelling of the complement's role, providing not only the chance for individualized post-transplant recurrence risk assessment, but also the possibility of a highly effective treatment through pharmacological C5-9 blockade with eculizumab. The overall outcome and prognosis of patients with aHUS has dramatically improved since the approval of this drug in 2011, allowing renal transplant to be a much safer option for these patients. Our aim was to present a proposal for the management of patients with aHUS, candidates for renal transplantation, in the light of the most recent studies.

Keywords: Atypical hemolytic uremic syndrome, complement dysregulation, eculizumab, kidney transplantation

(C) 2021 Portuguese Journal of Nephrology \& Hypertension. Published by Publicações Ciência \& Vida This is an open access article under the CC BY-NC-ND license (http://creativecommons.org/licenses/by-nc-nd/4.0/).

\section{INTRODUCTION}

Thrombotic microangiopathies (TMA) include a group of entities presenting with microvascular endothelial lesion and thrombosis ${ }^{1}$.

Among TMAs, atypical hemolytic uremic syndrome (aHUS) is one of the most challenging diseases for a nephrologist, with high rates of progression to end-stage renal disease (ESRD) and post-transplant recurrence ${ }^{2}$. It is an ultra-rare disease, with an estimated prevalence in Europe of 2:1000000 adults ${ }^{1-3}$. Genetically determined or acquired complement dysregulation has been found in up to $70 \%$ of cases $^{2}$. This dysregulation may be hereditary, caused by mutations in genes that encode complement regulatory proteins, Factor $\mathrm{H}(\mathrm{CFH})$, Factor I (CFI), membrane cofactor protein (MCP), complement 3 (C3), Factor $B(C F B)$, thrombomodulin (THDB) or diglycerol kinase-E (DKGE), or acquired, in the presence of anti-FH antibody resulting in activation of the complement system ${ }^{1,4,5}$. In our paper we will use the term aHUS to designate complement mediated HUS.

Over the last few years, the knowledge of the pathogenesis of aHUS has greatly increased, with the unravelling of the complement's role, providing not only the chance for individualized post-transplant recurrence risk assessment, but also the possibility of a highly effective treatment through pharmacological C5-9 blockade bl, $6,7^{\text {. The overall }}$ outcome and prognosis of patients with aHUS has dramatically improved since the approval of eculizumab in 2011, a humanized monoclonal antibody that neutralizes complement protein $\mathrm{C} 5$, the only FDA-approved treatment for $\mathrm{aHUS}^{8}$. This drug has allowed renal transplant to be a much safer option for these patients.

The Portuguese consensus document was published in 2018 and since then several studies have been published, specifically concerning the renal transplant in this population $2,6,8,9$.

Our aim was to present a proposal for the management of patients with aHUS, candidates for renal transplantation, in the light of the most recent studies.

\section{PRE-TRANSPLANT EVALUATION}

All patients with aHUS that develop ESRD are potential candidates for renal transplantation and, as such, should undergo a thorough evaluation in order to determine risk of recurrence of the disease in the post-transplant period and ascertain an individualized eculizumab administration strategy.

In addition to the general pre-transplant evaluation, transversal to all candidates, aHUS patients in particular must meet certain criteria in order to be included on the waiting list for kidney transplantation. 


\section{Stabilization of the disease}

As kidney function may recover even months following eculizumab administration, it is recommended to wait at least 6 months to assure the irreversibility of kidney injury ${ }^{3,10}$. All extra-renal manifestations of the disease, including hematological alterations, must be resolved prior to inclusion on the transplant waiting list ${ }^{3,11}$.

\section{Evaluation of recurrence risk following kidney transplant}

For this purpose, patients with the diagnosis of aHUS, or patients that develop ESRD in the context of secondary TMA or gestational TMA, must have a complete blood sample analysis ${ }^{8}$, including:

- Serum antigenic levels: C3, CFB, CFH and CFI;

- Screening for FH autoantibodies;

- Cell surface expression of MCP by flow cytometry;

- Genetic testing for complement regulatory proteins: CFH, CFB,

$\mathrm{CFI}, \mathrm{C} 3, \mathrm{MCP}, \mathrm{TBDH}, \mathrm{DGKE}$ and $\mathrm{CFH}$ related proteins (CFHRP);

All the abovementioned tests are available in Centro Hospitalar e Universitário de Coimbra, except flow cytometry for MCP, which is presently being introduced and will be available in the foreseeable future.

\section{- Patient stratification according to risk of recurrence}

The risk of recurrence is mainly determined by the underlying genetic anomalies or, in patients with aHUS related to anti-FH antibodies, by the titer of those antibodies ${ }^{11,12}$. Patients with anomalies in the genes that code for complement circulating factors $\mathrm{CFH}, \mathrm{CFI}, \mathrm{C} 3$ and CFB have a high risk of recurrence $(75-90 \%, 45-80 \%, 50-60 \%$ and $40-70 \%$, respectively), as the complement protein defects (either in quantity or function) persist after transplantation. Patients with anomalies in either intra or transcellular proteins (MCP or DGKE) have a low recurrence risk as the transplanted organ usually expresses the mentioned proteins ${ }^{8,11-13}$. In patients in whom no mutations in the complement regulating proteins are identified, recurrence risk seems to be lower (30\%), but since studies are still scarce, these patients should be considered at moderate risk ${ }^{12}$.

\section{Table 1}

Patient stratifications according to recurrence risk following renal transplantation.

\begin{tabular}{c||l} 
Risk of recurrence & \multicolumn{1}{c}{ Criteria } \\
\hline High risk & - Previous aHUS recurrence in a former kidney transplant \\
& - Pathogenic variants of CFH, C3 and CFB \\
& - Risk polymorphisms in CFHRP \\
Moderate risk & - Pathogenic variants of CFI \\
& - Presence of anti-FH antibodies \\
& - Absence of genetic alterations or presence of alterations of \\
& undetermined significance \\
Low risk & - Isolated pathogenic variants of MCP or DGKE \\
& - Persistently negative anti-FH antibodies in the absence of other \\
& complement genetic study alterations
\end{tabular}

Taking this into account, patients can be stratified in three groups according to risk of recurrence (Table 1 ).

\section{Complement inhibitory medication}

Eculizumab may be used either as prophylactic therapy or as a rescue therapy.

Three observations have prompted the use of prophylaxis, instead of therapy for overt recurrence: 1 ) aHUS recurrence usually occurs very early in the post-transplant course 12,$13 ; 2$ ) the benefits of eculizumab in the recovery of renal function are more pronounced in patients who have not received a transplant than in the recipients of a kidney transplant $2,9,14,15 ; 3)$ "cryptic aHUS", defined as the progression of a TMA process in the absence of the hallmark hematologic features, is increasingly recognized, and may lead to irreversible renal injury discovered late during the process $^{16-18}$.

In agreement with these observations, recent studies have suggested that the pretransplant initiation of eculizumab resulted in both a lower clinical and subclinical recurrence rate and in a better allograft function than that of the post-transplant initiation of eculizumab ${ }^{2,9}$. In one of the largest published series, with 126 renal transplants in patients with aHUS, only one patient out of the 52 submitted to prophylactic eculizumab (34 in the high-risk group) showed recurrence of the disease, occurring after eculizumab discontinuation. On the other hand, from the 74 patients not medicated with prophylactic eculizumab (35 in the high risk group and 30 in the moderate risk group), 39 (52.6\%) developed disease recurrence ${ }^{2}$.

However, despite its outstanding results, the high cost of eculizumab has led to the rise of more restrictive use strategies, trying to identify patients in which it can be reserved for recurrence situations rather than as a preemptive medication ${ }^{2,8}$. In line with this premise, latest recommendations, including the 2017 KDIGO guidelines, advocate the use of prophylactic complement blockade based on the recurrence risk ${ }^{17,19,20}$

In high risk and moderate risk patients, prophylactic administration is recommended, as studies show a similar recurrence ratio between these 2 groups $^{2}$. In the moderate risk group, the decision may be individualized for each patient but, if decided not to give prophylactically, eculizumab should be approved for these patients before placement on the waiting list for renal transplantation, because prompt initiation of eculizumab $(<24-48 \mathrm{H})$ in case of recurrence is critical in defining subsequent graft survival. For low-risk patients, prophylaxis is not recommended.

The ideal prophylactic scheme with eculizumab is not well defined. Available recommendations propose the following 8 :

- 24h before transplantation (living donor) or immediately before transplantation (deceased donor) - $900 \mathrm{mg}$ intravenous (iv)

- Days 7, 14 and 21 post-transplant - $900 \mathrm{mg}$ iv

- Every two weeks from week 5-1200 mg iv 
Of note, in patients whose only alteration is anti-FH antibodies persistence, especially if in high titers $(>1000 \mathrm{AU} / \mathrm{ml}$, reference $<200$ $\mathrm{AU} / \mathrm{ml}$ ), therapy with a cell depleting agent, such as rituximab, should be pondered, in order to attain negative antibody titers previous to transplant.

\section{Immunization}

All patients with aHUS candidates for renal transplantation should get anti-meningococcal and anti-pneumococcal immunization before entering the waiting list and at least two weeks before transplantation, even if prophylactic eculizumab is not used. The Advisory Committee on Immunization Practices recommends simultaneous immunization with MenACWY (with booster doses every 5 years) and MenB ${ }^{21}$. As adolescents and young adults are the primary carriers of meningococci, vaccination of susceptible close contacts (including siblings and parents) can decrease meningococcal carriage and transmission and may be considered ${ }^{22}$.

\section{It is also recommended to get annual influenza immunization.}

\section{Living donor evaluation}

Historically, renal transplantation with a living donor was associated with a high recurrence risk. Moreover, the nephrectomy could trigger TMA in the genetically susceptible donor ${ }^{8}$. However, knowledge of the pathophysiology and the introduction of eculizumab have changed this paradigm and turned this into a valid option, considered with caution on a case-by-case basis.

A study by Bresin and colleagues reported higher 1-year graft survival for living donor transplants compared with cadaveric-donor transplants ( $50 \%$ vs $32 \%$ ), data concordant with the UNOS Renal Transplant Registry data, where living-donor transplantation was associated with 1 -year graft survival of $93 \%$ vs $87 \%$ in deceased-transplant ${ }^{13,23}$. This difference may be partly explained by complement activation following prolonged cold ischemia time.

Both potential donor and receptor should participate in decision making after they have understood the risks and benefits of this option.

The possibility of living donor should respect some criteria, and is contraindicated in specific situations ${ }^{3}$ :

- Related donor, if the mutation in the receptor is not identified or is uncertain or if the donor has the same mutation as the receptor;

- If the receptor presents anti-donor specific antibodies.

\section{PERI-OPERATIVE MANAGEMENT}

In the setting of renal transplantation, prolonged cold ischemia time ( $>8 \mathrm{~h}$ ) should be avoided, as should be donors after cardiac death and young deceased donors ( $<30$ years old). It is however important to mention that as kidney transplantation is dependent on the availability of donors, these do not constitute contraindications ${ }^{24}$. Also, to minimize the burden of endothelial-damaging factors that trigger the disease onset, blood pressure must be strictly controlled. Induction immunosuppression should be individualized according to the patient's immunological risk. As for maintenance immunosuppression, mTOR inhibitors should be avoided, as they have been shown to be an independent risk factor for the development of TMA. Even though treatment with calcineurin inhibitors (CNI) is associated with post-transplant TMA, studies have shown no reduction in the risk of aHUS recurrence with $\mathrm{CNI}$ avoidance. Therefore, low-dose calcineurininhibitors (targeting 20-30\% lower-than-usual trough levels) should be preferentially used ${ }^{3,8,13,25}$. However, since aHUS patients are prone to hypersensitizing events, such as blood transfusions and previous transplants, the risk of rejection versus the risk aHUS recurrence must be carefully balanced when adjusting immunosuppression.

\section{POST-TRANSPLANT MANAGEMENT}

\section{Clinical monitoring}

In the post-transplant period, factors such as surgical aggression, ischemia-reperfusion injury, antibody-mediated acute rejection, use of immunosuppressants (particularly calcineurin inhibitors and mTOR inhibitors) and infections (especially those caused by cytomegalovirus, influenza virus, parvovirus B19, BK virus, respiratory and gastrointestinal infections) can trigger endothelial injury ${ }^{24,26}$.

The risk of recurrence is higher in the first year post-transplant, and $60 \%$ of cases recur in the first month following transplantation. Follow-up of these patients must be exhaustive, and it is important to promptly identify any disease manifestations ${ }^{1,27}$ (Table 2 ):

In the series published by Zuber et $\mathrm{al}^{2}, 12 \%$ of patients who did not receive preemptive eculizumab developed some degree of graft dysfunction. This highlights the importance of maintaining a high degree of suspicion and a low threshold for biopsy. Furthermore,

\section{Table 2}

Clinical manifestations of aHUS recurrence in the post-transplant period.

\begin{tabular}{|c|c|}
\hline End-organ & Manifestations \\
\hline Renal & $\begin{array}{l}\text { Acute kidney injury, arterial hypertension, proteinuria, active uri- } \\
\text { nary sediment }\end{array}$ \\
\hline Hematologic & Hemolytic anemia, thrombocytopenia \\
\hline Neurologic & $\begin{array}{l}\text { Encephalopathy, convulsions, irritability, drowsiness, hemiplegia, } \\
\text { stupor, coma }\end{array}$ \\
\hline Gastrointestinal & $\begin{array}{l}\text { Diarrhea, nausea, vomiting, hepatic cytolysis, pancreatitis, intes- } \\
\text { tinal bleeding }\end{array}$ \\
\hline Cardiovascular & Myocardial infarction, myocarditis, heart failure, cardiomyopathy \\
\hline Peripheral vascular & Gangrenous lesions in fingers and toes \\
\hline Pulmonary & Pleural effusion, pulmonary hemorrhage \\
\hline Ocular & Diplopia, cortical blindness \\
\hline Skeletal muscle & Rhabdomyolysis \\
\hline Cryptic aHUS & Graft dysfunction without other manifestations \\
\hline
\end{tabular}


studies show that profound systemic hemolysis can be lacking in $\leq$ $60 \%$ of patients with complement mediated $\mathrm{TMA}^{28}$. We believe in these patients protocoled biopsies should be considered, particularly if complement blockade is not performed preemptively.

\section{Laboratory monitoring}

Among aHUS patients, hemoglobin, platelet count, lactate dehydrogenase (LDH) and serum creatinine should be closely monitored. In patients with suspicion of recurrence, additional analysis including peripheral blood smear, haptoglobin, urine analysis with sediment analysis and urinary protein/creatinine ratio should also be performed $^{8}$.

In patients treated with eculizumab, we measure total hemolytic complement ( $\mathrm{CH} 50$ ) to assess the effectiveness of complement blockade: patients with complete blockade should have $\mathrm{CH} 50<10 \%$. If available, trough serum eculizumab levels can be measured with a target level of $>100 \mathrm{ug} / \mathrm{ml}^{8,29}$. A promising method which can contribute to guide eculizumab therapeutic is ex vivo C5b9 formation ${ }^{28}$. Timmermans et al recently described massive ex vivo $\mathrm{C} 5 \mathrm{~b}$ 9 formation in patients with complement mediated aHUS, either primary or with coexisting conditions (such as hypertensive emergency, pregnancy or de novo TMA after kidney transplantation), and also found that patients treated with eculizumab that showed persistent inhibition of ex vivo C5b9 formation, even if classical pathway functional activity $>10 \%$, did not experience a relapse of the disease despite a prolonged interdose interval ${ }^{28}$.

Proposed post-transplant monitoring of $\mathrm{CH} 50$ in patients treated with eculizumab is daily in the immediate post-transplant period (during hospitalization), with monitoring before eculizumab administration in the first 4 doses, weekly up to 6 months, biweekly from 6 to 12 months and then monthly after 12 months.

\section{Antibiotic prophylaxis}

In patients receiving eculizumab, there is a debate concerning whether to use or not antibiotic prophylaxis for meningococcal infection. If the patient has received the recommended immunizations before the transplantation, there may be no need. However, if there is not enough time to wait for the immune response, appropriate antibiotics (penicillin or fluoroquinolone) should be used for at least 14 days $^{8}$.

Nevertheless, patients should be alert to the signs and symptoms of the infection and for when to seek medical consultation.

\section{Disease recurrence}

\section{Patients treated with eculizumab}

We found 6 cases in the literature which developed thrombotic microangiopathy (TMA) in the post-transplant period while on eculizumab prophylaxis (27-32). In one case, the patient lost the graft due to TMA with renal artery thrombosis ${ }^{30}$. In the remaining 5 cases the dose of eculizumab was increased with different strategies: 1) increase maintenance dose from $1200 \mathrm{mg}$ to $1500 \mathrm{mg}$ every two weeks ${ }^{27,29}$; 2) repeat induction doses of eculizumab (900 mg weekly for 4 weeks or $600 \mathrm{mg}$ weekly for 1 week in a pediatric patient, followed by maintenance doses) ${ }^{28,31}$; 3) decrease interval of administration after its prolongation ( $1200 \mathrm{mg}$ every three weeks to every two weeks) ${ }^{32}$. All these strategies were successful.

We found no cases of plasma exchange use in this situation.

\section{Patients not treated with eculizumab}

Treatment of aHUS in the kidney transplant patient should obey the same principles as in every other patient, including platelet transfusion if necessary, fluid and electrolyte management, avoidance of nephrotoxic medications and blood pressure control ${ }^{1}$.

In a recent study, eculizumab therapy for aHUS recurrence significantly improved death-censored graft survival when compared with patients treated with plasmapheresis alone, particularly if started < 1 week after diagnosis ${ }^{2}$.

\section{Eculizumab available}

If the patient presents clinical or laboratory manifestations compatible with TMA or if a graft biopsy reveals findings in accordance with this diagnosis, rescue therapy with eculizumab should be started, ideally in the first 24-48h following diagnosis, as the recovery of renal graft function critically depends on the timing of eculizumab administration ${ }^{1,17}$.

Proposed scheme is intravenous administration of $900 \mathrm{mg}$ iv weekly for the first 4 weeks, followed by 1200 mg every 2 weeks from week 5. In patients receiving plasmapheresis, a supplemental dose is administered after each plasma exchange ${ }^{8}$.

\section{Eculizumab not available}

In cases when eculizumab is not approved and available (situation that should only occur in the context of de novo TMA diagnosis posttransplant), plasmapheresis must be performed as a bridge for eculizumab (Table 3).

\section{$\underline{\text { Table } 3}$}

Plasmapheresis scheme for the treatment of aHUS recurrence.

\begin{tabular}{l} 
Plasmapheresis \\
\hline - Plasma volume: $60-75 \mathrm{ml} / \mathrm{kg} / \mathrm{session}$ \\
- Reposition with fresh frozen plasma \\
- Daily until normalization of platelet count, LDH and hemoglobin and until sustained \\
improvement in graft function or until availability of eculizumab
\end{tabular}

\section{Efficacy control}

Hematological parameters (hemoglobin, platelet count, peripheral blood smear, LDH and haptoglobin), renal function (serum creatinine 
and urinary output) should be monitored. Before each administration of eculizumab, CH50 levels should be measured (value $>10 \%$ can be an indicator to increase the dosage).

\section{Eculizumab discontinuation}

Information about eculizumab withdrawal after transplantation is very scarce and as such, the ideal duration of eculizumab therapy in kidney transplant patients is not yet established ${ }^{6}$. Reported relapse rates in aHUS after stopping eculizumab are as high as $30 \%$, suggesting indefinite therapy is reasonable and that patients who stop should be closely monitored ${ }^{33}$. Small series and in vitro data suggest that prolonging intervals between doses of eculizumab based on $\mathrm{CH} 50$ results may be safe ${ }^{34,35}$.

- In high-risk patients, therapy should be continued ad eternum (or at least should not be discontinued in the first two years). The frequency of administration can, however, be reduced, tapered according to the degree of complement suppression (CH50 should be kept < 10\%).

- In moderate-risk patients, eculizumab discontinuation can be considered after a long period without active disease; in this case, a close follow-up should be kept. A suggestion would be to slowly reduce the frequency of administration, aiming for a period of partial complement suppression $(\mathrm{CH} 50<30 \%)$ before complete withdrawal. In these patients, hemoglobin, platelets, serum creatinine and LDH should be monitored in the following schedule after stopping the drug: at 2 and 4 weeks, then monthly for 6 months, then every 3-4 months ${ }^{33}$.

- In patients with aHUS recurrence, given the high risk of graft loss, recommendations are the same for the high-risk group.

\section{CONCLUSION}

Recent advances in both the physiopathology and treatment of aHUS, specifically the introduction of complement blockade with eculizumab, have led to a revolution in renal transplantation outcomes in these patients, making renal transplantation (including living donor) a safe and suitable option. The best available evidence suggests that eculizumab should be administered preemptively in all high risk, and, ideally, in all medium-risk patients. Duration of eculizumab therapy following renal transplant is still not completely clear. Under close surveillance, it seems reasonable to titrate dose according to $\mathrm{CH} 50$ levels in high- and medium-risk patients; in case of relapse, eculizumab therapy should be maintained indefinitely.

Disclosure of potential conflicts of interest: none declared.

\section{References}

1. Raina R, Krishnappa V, Blaha T, Kann T, Hein W, Burke L, et al. Atypical hemolytic-uremic syndrome: an update on pathophysiology, Diagnosis, and Treatment. Ther Apher Dial Off peer-reviewed I Int Soc Apher Japanese Soc Apher Japanese Soc Dial Ther. 2019 Feb;23(1):4-21.

2. Zuber J, Frimat M, Caillard S, Kamar N, Gatault P, Petitprez F, et al. Use of highly individualized complement blockade has revolutionized clinical outcomes after kidney transplantation and renal epidemiology of atypical hemolytic uremic syndrome. J Am Soc Nephrol. 2019 Dec;30(12):24492463.

3. Azevedo A, Faria B, Teixeira C, Carvalho F, Neto G, Santos J, et al. Portuguese consensus document statement in diagnostic and management of atypical hemolytic uremic syndrome. Port J Nephrol Hypert. 2018;32(3):211-232.

4. Fakhouri F, Zuber J, Frémeaux-Bacchi V, Loirat C. Haemolytic uraemic syndrome. Lancet (London, England). 2017 Aug;390(10095):681-696.

5. Lemaire M, Frémeaux-Bacchi V, Schaefer F, Choi M, Tang WH, Le Quintrec M, et al. Recessive mutations in DGKE cause atypical hemolytic-uremic syndrome. Nat Genet. 2013 May;45(5):531536.

6. Wijnsma KL, Duineveld C, Wetzels JFM, van de Kar NCAJ. Eculizumab in atypical hemolytic uremic syndrome: strategies toward restrictive use. Pediatr Nephrol. 2019 Nov;34(11):2261-2277.

7. I. AP. Soliris: summary of product characteristics 2012.

8. Java A. Peri- and post-operative evaluation and management of atypical hemolytic uremic syndrome (aHUS) in kidney transplantation. Adv Chronic Kidney Dis. 2020 Mar;27(2):128-137.

9. Siedlecki AM, Isbel N, Vande Walle J, James Eggleston J, Cohen DJ. Eculizumab use for kidney transplantation in patients With a diagnosis of atypical hemolytic uremic syndrome. Kidney Int reports. 2019 Mar;4(3):434-446.

10. Povey $H$, Vundru R, Junglee $N$, Jibani M. Renal recovery with eculizumab in atypical hemolytic uremic syndrome following prolonged dialysis. Clin Nephrol. 2014 Nov;82(5):326-331.

11. Santos J, Oliveira N, Azevedo A, Fidalgo T, Neto G. 50 Perguntas chave na Nefrologia. No diagnóstico e terapêutica das microangiopatias trombóticas. 2019.

12. Le Quintrec M, Zuber J, Moulin B, Kamar N, Jablonski M, Lionet A, et al. Complement genes strongly predict recurrence and graft outcome in adult renal transplant recipients with atypical hemolytic and uremic syndrome. Am J Transplant Off J Am Soc Transplant Am Soc Transpl Surg. 2013 Mar;13(3):663-675.

\section{Figure 1}

Options for the treatment of aHUS with eculizumab. Adapted from6. Option A represents the current standard regimen, with ad eternum biweekly administration. Options B-E illustrate more restrictive use.

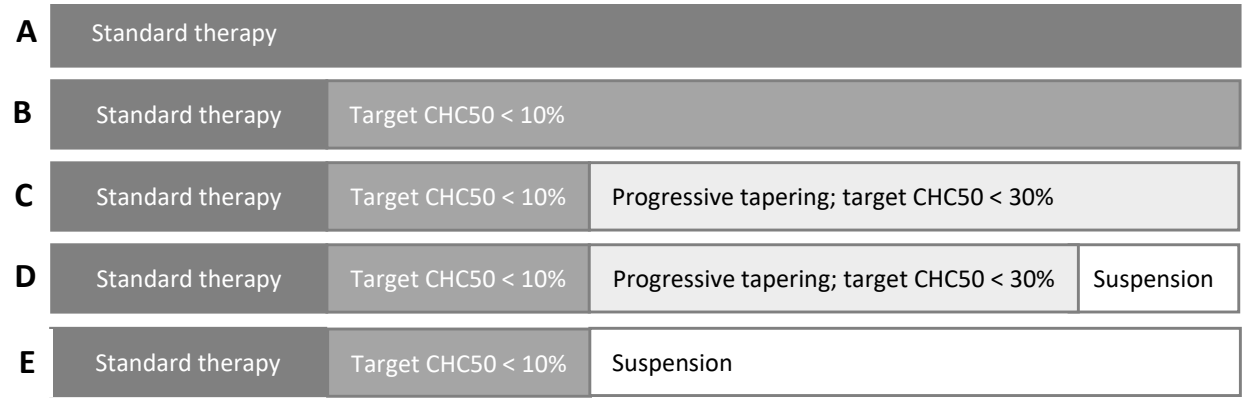

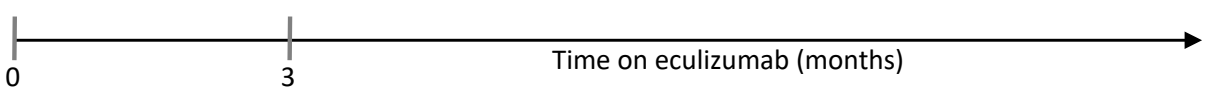


13. Bresin E, Daina E, Noris M, Castelletti F, Stefanov R, Hill P, et al. Outcome of renal transplantation in patients with non-Shiga toxin-associated hemolytic uremic syndrome: prognostic significance of genetic background. Clin J Am Soc Nephrol. 2006 Jan;1(1):88-99.

14. Licht C, Greenbaum LA, Muus P, Babu S, Bedrosian CL, Cohen DJ, et al. Efficacy and safety of eculizumab in atypical hemolytic uremic syndrome from 2-year extensions of phase 2 studies. Kidney Int. 2015 May;87(5):1061-1073.

15. Fakhouri F, Hourmant M, Campistol JM, Cataland SR, Espinosa M, Gaber AO, et al. Terminal complement inhibitor eculizumab in adult patients With atypical hemolytic uremic syndrome: a single-arm, open-label trial. Am J kidney Dis Off J Natl Kidney Found. 2016 Jul:68(1):84-93.

16. Belingheri M, Possenti I, Tel F, Paglialonga F, Testa S, Salardi S, et al. Cryptic activity of atypical hemolytic uremic syndrome and eculizumab treatment. Pediatrics. 2014 Jun;133(6):e1769-e1771.

17. Zuber J, Fakhouri F, Roumenina LT, Loirat C, Frémeaux-Bacchi V. Use of eculizumab for atypical haemolytic uraemic syndrome and C3 glomerulopathies. Nat Rev Nephrol. 2012 Nov;8(11):643657.

18. Legendre CM, Licht C, Muus P, Greenbaum LA, Babu S, Bedrosian C, et al. Terminal complement inhibitor eculizumab in atypical hemolytic-uremic syndrome. N EngI J Med. 2013 Jun;368(23):21692181.

19. Goodship THJ, Cook HT, Fakhouri F, Fervenza FC, Frémeaux-Bacchi V, Kavanagh D, et al. Atypical hemolytic uremic syndrome and C3 glomerulopathy: conclusions from a "Kidney Disease: Improving Global Outcomes" (KDIGO) Controversies Conference. Vol. 91, Kidney international. United States; 2017:539-551.

20. Campistol JM, Arias M, Ariceta G, Blasco M, Espinosa L, Espinosa M, et al. An update for atypical haemolytic uraemic syndrome: diagnosis and treatment. A consensus document. Nefrologia. 2015;35(5):421-447.

21. Cohn AC, MacNeil JR, Clark TA, Ortega-Sanchez IR, Briere EZ, Meissner HC, et al. Prevention and control of meningococcal disease: recommendations of the Advisory Committee on Immunization Practices (ACIP). MMWR Recomm reports Morb Mortal Wkly report Recomm reports. 2013 Mar;62(RR-2):1-28.

22. Burman C, Serra L, Nuttens C, Presa J, Balmer P, York L. Meningococcal disease in adolescents and young adults: a review of the rationale for prevention through vaccination. Hum Vaccin Immunother. 2019;15(2):459-469.

23. Cecka JM. The UNOS Scientific Renal Transplant Registry. Clin Transpl. 1999;1-21.

24. Zuber J, Le Quintrec M, Morris H, Frémeaux-Bacchi V, Loirat C, Legendre C. Targeted strategies in the prevention and management of atypical HUS recurrence after kidney transplantation. Transplant Rev (Orlando). 2013 Oct;27(4):117-125.
25. Duineveld C, Verhave JC, Berger SP, van de Kar NCAJ, Wetzels JFM. Living donor kidney transplantation in atypical hemolytic uremic syndrome: a case series. Am J kidney Dis Off J Natl Kidney Found. 2017 Dec;70(6):770-777.

26. Zuber J, Le Quintrec M, Sberro-Soussan R, Loirat C, Frémeaux-Bacchi V, Legendre C. New insights into postrenal transplant hemolytic uremic syndrome. Nat Rev Nephrol. 2011 Jan;7(1):23-35.

27. Loirat C, Frémeaux-Bacchi V. Atypical hemolytic uremic syndrome. Orphanet J Rare Dis. 2011 Sep;6:60.

28. Timmermans SAMEG, Damoiseaux JGMC, Werion A, Reutelingsperger CP, Morelle J, van Paassen P. Functional and genetic landscape of complement dysregulation along the spectrum of thrombotic microangiopathy and its potential implications on clinical outcomes. Kidney Int reports. 2021 Apr;6(4):1099-1109.

29. Wong E, Challis R, Sheerin N, Johnson S, Kavanagh D, Goodship THJ. Patient stratification and therapy in atypical haemolytic uraemic syndrome (aHUS). Immunobiology. 2016 Jun;221(6):715718.

\section{ORCID}

Ana Carolina Figueiredo iD 0000-0002-5224-2330

Nuno Afonso Oliveira iD 0000-0002-4112-9236

Catarina Romãozinho (iD) 0000-0002-5526-9336

Helena Sá (iD) 0000-0001-5231-2629

Arnaldo Figueiredo (iD) 0000-0002-7157-0081

Rui Alves (iD) 0000-0003-3922-3618

\section{Correspondence to:}

Ana Carolina Figueiredo

Av. Bissaya Barreto, no 299, 3000-076 Coimbra, Portugal

Email: carolinarfigueiredo@gmail.com 